\title{
GLOBAL WELLPOSEDNESS FOR KDV BELOW $L^{2}$
}

\author{
J. Colliander, G. Staffilani, and H. Takaoka
}

\begin{abstract}
The initial value problem for the Korteweg-deVries equation on the line is shown to be globally wellposed for rough data. In particular, we show global wellposedness for certain initial data in $H^{s}$ for an interval of negative $s$. The proof is an adaptation of a general argument introduced by Bourgain to prove a similar result for a nonlinear Schrödinger equation. The proof relies on a generalization of the bilinear estimate of Kenig, Ponce and Vega.
\end{abstract}

\section{Introduction}

Consider the initial value problem for the Korteweg-deVries (KdV) equation

$$
\left\{\begin{array}{c}
\partial_{t} u+\partial_{x}^{3} u+\frac{1}{2} \partial_{x} u^{2}=0 \\
u(0)=\phi \in H^{s}(\mathbb{R}), \quad-\frac{3}{4}<s<0
\end{array}\right.
$$

The local wellposedness theory in [1], [6] shows, for $s>-\frac{3}{4}$, there exists a unique solution $u \in C\left([0, T] ; H^{s}(\mathbb{R})\right)$ which depends continuously on the data $\phi$. The lifetime $T$ is bounded below by a negative power of $\|\phi\|_{H^{s}}$. The problem is said to be globally wellposed if we can take $T=\infty$. Iterating the local result naturally extends the existence interval. However, successive time steps may shrink due to the growth of $\|u(t)\|_{H^{s}}$. Consequently, the iteration process may only extend the solution to a longer, but still finite, existence interval.

Solutions of (1.1) satisfy,

$$
\|u(t)\|_{L^{2}}=\|\phi\|_{L^{2}} .
$$

Since (1.2) prevents the growth of $\|u(t)\|_{L^{2}}$, successive steps in the iteration of the local result are all greater than a fixed size. Therefore, for $s \geq 0,(1.1)$ is globally wellposed. Our first main result shows (1.1) is globally wellposed for initial data having a certain degree of regularity below $L^{2}$. It is conjectured that $\mathrm{KdV}$ is globally wellposed in $H^{s}(\mathbb{R})$ for $s>-\frac{3}{4}$ but we do not obtain this full range of $s$.

The proof is an adaptation of the general method introduced by Bourgain [3] to establish a similar global wellposedness result for the $2 d$ cubic nonlinear Schrödinger equation below $H^{1}$. Fonseca, Linares and Ponce [4] used Bourgain's approach to prove $\mathrm{mKdV}$ (replace $u^{2}$ by $u^{3}$ in (1.1)) is globally wellposed below $H^{1}$. Similar results for nonlinear wave equations have been obtained by Bourgain [2] and by Keel and Tao [5]. Takaoka [7] and Tzvetkov [8] have obtained global results below $L^{2}$ for the $K P-I I$ equation using similar methods.

Received September 23, 1998. Revised November 1, 1999. 
The idea is to exploit the $L^{2}$ conservation law (1.2) of (1.1) even though the data $\phi \notin L^{2}$. We briefly describe the proof.

Fix $T>0$. We construct the solution $u$ of $(1.1)$ on $[0, T]$. Since $T$ is arbitrary, this implies global existence; global wellposedness then follows from local wellposedness.

Break the data $\phi$ into low and high frequency pieces by writing

$$
\phi=\phi_{0}+\psi_{0}, \quad \phi_{0}=\mathbb{P}_{N} \phi,
$$

where $\mathbb{P}_{N}$ is the Dirichlet projection defined by

$$
\widehat{\mathbb{P}_{N} \phi}=\chi_{[-N, N]} \widehat{\phi}
$$

and $\chi_{[-N, N]}$ is the characteristic function of the interval $[-N, N]$ and $\widehat{\phi}$ denotes the Fourier transform of $\phi$. The number $N$ will be selected later, $N=N(T)$.

Let $\phi_{0}$ evolve forward to $u_{0}$ according to $\mathrm{KdV}$,

$$
\left\{\begin{array}{c}
\partial_{t} u_{0}+\partial_{x}^{3} u_{0}+\frac{1}{2} \partial_{x} u_{0}^{2}=0, \quad x \in \mathbb{R} \\
u_{0}(0)=\phi_{0}
\end{array}\right.
$$

Let $\psi_{0}$ evolve according to

$$
\left\{\begin{array}{c}
\partial_{t} v_{0}+\partial_{x}^{3} v_{0}+\frac{1}{2} \partial_{x}\left(v_{0}^{2}+2 u_{0} v_{0}\right)=0, \quad x \in \mathbb{R} \\
v_{0}(0)=\psi_{0} .
\end{array}\right.
$$

The equation (1.6) is selected so that

$$
u(t)=u_{0}(t)+v_{0}(t)
$$

solves (1.1). We may sometimes refer to (1.6) as the "difference equation" since its evolution expresses the difference between $u$ and $u_{0}$. The initial value problems $(1.5),(1.6)$ are shown to be locally wellposed on a $\delta$-sized time interval with

$$
\delta=\delta\left(\left\|\phi_{0}\right\|_{L^{2}}\right)
$$

provided $N$ is sufficiently large. Since $\phi_{0} \in L^{2}$, the decomposition (1.3) gives $\delta=\delta(N)$. Split $v_{0}$ by writing

$$
v_{0}(t)=e^{-t \partial_{x}^{3}} \psi_{0}+w_{0}(t) .
$$

Define

$$
\phi_{1}=u_{0}(\delta)+w_{0}(\delta) ; \quad \psi_{1}=e^{-\delta \partial_{x}^{3}} \psi_{0}
$$

and evolve $\phi_{1} \longmapsto u_{1}, \psi_{1} \longmapsto v_{1}$ according to

$$
\begin{gathered}
\left\{\begin{array}{c}
\partial_{t} u_{1}+\partial_{x}^{3} u_{1}+\frac{1}{2} \partial_{x} u_{1}^{2}=0 \\
u_{1}(0)=\phi_{1}
\end{array}\right. \\
\left\{\begin{array}{c}
\partial_{t} v_{1}+\partial_{x}^{3} v_{1}+\frac{1}{2} \partial_{x}\left(v_{1}^{2}+2 u_{1} v_{1}\right)=0 \\
v_{1}(0)=\psi_{1} .
\end{array}\right.
\end{gathered}
$$

We wish to show (1.11), (1.12) are locally wellposed on an interval of the same size $\delta$. This requires $\left\|\phi_{1}\right\|_{L^{2}} \sim\left\|\phi_{0}\right\|_{L^{2}}$ which follows from an estimate 
on $\left\|w_{0}(\delta)\right\|_{L^{2}}$. Since $w_{0}(t)$ is part of the evolving solution of (1.12) with data $\psi_{0} \notin L^{2}$, it is not obvious that $w_{0}(\delta) \in L^{2}$. The process (1.9)-(1.12) may be iterated with the same sized $\delta$ throughout until, say

$$
\left\|w_{0}\right\|_{L^{2}}+\cdots+\left\|w_{M}\right\|_{L^{2}} \sim\left\|\phi_{0}\right\|_{L^{2}} ; \quad M=M(N) .
$$

This extends the solution to an interval of size $M \delta$. The final step is to show that, for appropriate $s$, we can choose $N$ large enough to guarantee

$$
M \delta>T \text {. }
$$

The main new technical ingredient is the observation that the bilinear estimate of Kenig, Ponce and Vega [6],

$$
\left\|\partial_{x} u v\right\|_{X_{s, b-1}} \leq C\|u\|_{X_{s, b}}\|v\|_{X_{s, b}}, \quad s>-\frac{3}{4}, \quad b \gtrsim \frac{1}{2},
$$

can be strengthened in the case $s=0$ to a variant of the false inequality

$$
\left\|\partial_{x}(u v)\right\|_{X_{0, b-1}} \leq C\|u\|_{X_{\gamma, b}}\|v\|_{X_{\gamma, b}}
$$

for $\gamma<0$. The two parameter spaces $X_{s, b}$ are defined in (1.17) below. As stated, (1.16) is not true (see Proposition 1 in Section 2 below.) However, (1.16) does hold provided $u$ and $v$ have their spatial Fourier transforms supported away from zero. This estimate reveals "extra" smoothing, beyond the recovery of the derivative, which allows us to show $w_{0}(\delta) \in L^{2}$. The failure of (1.16) occurs due to interactions with low frequencies and motivates us to consider initial data in the class $H^{0, a}$ defined below.

A preprint containing similar results to those found here was circulated by the first two authors last year. However, the failure of (1.16) was not accounted for and invalidates the claims made in that preprint. The first two authors thank Professor Y. Tsutsumi for kindly pointing out the issues arising from low frequency interactions. We thank C. Kenig for several nice discussions.

The rest of the paper is organized as follows. This section concludes with some definitions and a statement of the main result. Section 2 addresses generalizations of the bilinear estimate (1.15). Local wellposedness of the problems (1.5), (1.6) is shown in section 3. The final section contains the proof of global wellposedness.

Notation and statement of result. We recall the definition of the spaces $X_{s, b}$. These spaces, introduced in [1], are tailored to the linear part of the KdV equation. We will prove a family of bilinear estimates which generalizes the sharp estimate of [6] in the next section.

We will write $a+$ for $a \in \mathbb{R}$ to mean $a+\epsilon$ for a sufficiently small $\epsilon>0$. We will also write $\int_{*}$ to denote the convolution integral $\underset{\substack{k=k_{1}+k_{2} \\ \lambda=\lambda_{1}+\lambda_{2}}}{\int}$ in various places below. 
Let $X_{s, b}$ denote the closure of the Schwarz class $\mathcal{S}\left(\mathbb{R}^{2}\right)$ in the norm

$$
\|u\|_{X_{s, b}}=\left(\int(1+|k|)^{2 s}\left(1+\left|\lambda-k^{3}\right|\right)^{2 b}|\widehat{u}(k, \lambda)|^{2} d k d \lambda\right)^{\frac{1}{2}} .
$$

Let $X_{s, b}^{\delta}$ denote the closure of $\mathcal{S}\left(\mathbb{R}^{2}\right)$ in the norm

$$
\|u\|_{X_{s, b}^{\delta}}=\inf \left(\int(1+|k|)^{2 s}\left(1+\left|\lambda-k^{3}\right|\right)^{2 b}|\widehat{w}(k, \lambda)|^{2} d k d \lambda\right)^{\frac{1}{2}}
$$

where the infimum is taken over all functions $w$ such that $w(t)=u(t)$ for $t \in$ $[-\delta, \delta]$. When $b>\frac{1}{2}, u \in X_{s, b}^{\delta}$ implies $u \in C_{H^{s}(\mathbb{R})}([-\delta, \delta])$. We introduce a modification of the space $H^{s}(\mathbb{R})$ with a parameter which allows us to control low frequencies. Define $H^{s, a}(\mathbb{R})=\left\{\phi:\|\phi\|_{H^{s, a}(\mathbb{R})}<\infty\right\}$ where

$$
\|\phi\|_{H^{s, a}(\mathbb{R})}=\left(\int\left[(1+|k|)^{s} \chi_{\{|k| \geq 1\}}(k)+|k|^{a} \chi_{\{|k|<1\}}(k)\right]^{2}|\widehat{\phi}(k)|^{2} d k\right)^{\frac{1}{2}} .
$$

Correspondingly, we define $X_{s, a, b}$ and $X_{s, a, b}^{\delta}$ by replacing $(1+|k|)^{2 s}$ in $X_{s, b}$ and $X_{s, b}^{\delta}$ by $\left[(1+|k|)^{s} \chi_{\{|k| \geq 1\}}(k)+|k|^{a} \chi_{\{|k|<1\}}(k)\right]^{2}$.

Remark 1. For $a>-\frac{1}{2}, \mathcal{S}(\mathbb{R}) \subset H^{s, a}(\mathbb{R})$ and the space $H^{s, a}(\mathbb{R})$ may be defined as the norm-closure of $\mathcal{S}(\mathbb{R})$. For $a<-\frac{1}{2}$, there are Schwartz class functions which are not contained in $H^{s, a}(\mathbb{R})$ so some extra care is required in defining the space.

With these notions, our main result may be stated.

Theorem 1. The initial value problem

$$
\left\{\begin{array}{c}
\partial_{t} u+\partial_{x}^{3} u+\frac{1}{2} \partial_{x} u^{2}=0 \\
u(0)=\phi \in H^{s, a}(\mathbb{R})
\end{array}\right.
$$

is globally wellposed in $H^{s, a}$ for $s \in\left(s_{0}(a), 0\right]$ with $s_{0}(a)=\frac{a}{12}<0$ for appropriate $a<0$. Moreover, for $\phi \in H^{s, a}$ with $s \in\left(s_{0}(a), 0\right]$,

$$
u(t)-e^{-t \partial_{x}^{3}} \phi \in L^{2}(\mathbb{R}) \text { for all } t .
$$

Strictly speaking, Theorem 1 does not extend Bourgain's global result [1] for $L^{2}$ data since $H^{0, a}$ does not contain $L^{2}$ when $a<0$. As mentioned above, it is conjectured that the $\mathrm{KdV}$ equation is globally wellposed in $H^{s}(\mathbb{R}), s>-\frac{3}{4}$.

\section{Bilinear Estimate}

In this section, the following extension of the bilinear estimate in [6] is proven.

Theorem 2. The bilinear estimate

$$
\left\|\partial_{x} u v\right\|_{X_{\alpha, a, b-1}} \leq C\left(\|u\|_{X_{\gamma_{1}, a_{1}, b}}\|v\|_{X_{\gamma_{2}, a_{2}, \beta}}+\|u\|_{X_{\gamma_{1}, a_{1}, \beta}}\|v\|_{X_{\gamma_{2}, a_{2}, b}}\right),
$$


holds for parameters $\alpha, a, b, \gamma_{1}, a_{1}, \gamma_{2}$,

$$
b=\frac{1}{2}+, \quad \mu:=1-2 b+\beta, \quad \alpha, \gamma_{1}, \gamma_{2} \leq 0, \quad a, a_{1}, a_{2} \leq 0,
$$

and

$$
\begin{aligned}
-1 \leq\left(\alpha-\gamma_{i}\right) & \leq \min \left(2 \mu-\frac{1}{2},-a_{j} \frac{2 \mu-\frac{1}{2}}{\mu+\frac{1}{2}}\right), \quad i=1,2 \text { while } j=2,1, \\
-\left(\gamma_{1}+\gamma_{2}\right) & <\min \left(\frac{3}{2}+2 a, 2 \mu+\frac{1}{2}\right), \\
\alpha-\left(\gamma_{1}+\gamma_{2}\right) & <3 \mu-\frac{3}{4} .
\end{aligned}
$$

The reader may find it illuminating to verify that $\gamma_{1}=\gamma_{2}=\alpha=-\frac{3}{4}+$ satisfy the conditions above when we set $\mu=\frac{1}{2}, a=a_{1}=a_{2}=0$. Therefore, this theorem extends the bilinear estimate of [6]. The main point of the theorem is that the $\alpha=0$ case allows for $\gamma_{1}, \gamma_{2}<0$ provided the other parameters are selected appropriately.

Proof. The proof is a case-by-case analysis of various regions of the integral expression for $\left\|\partial_{x} u v\right\|_{X_{\alpha, a, b-1}}$. By duality, the object to be estimated may be rewritten

$$
\sup _{\|d\|_{L^{2}} \leq 1} \int_{*} \frac{(1+|k|)^{\alpha-a}|k|^{1+a} d(k, \lambda)}{\left(1+\left|\lambda-k^{3}\right|\right)^{b}} \widehat{u}\left(k_{1}, \lambda_{1}\right) \widehat{v}\left(k_{2}, \lambda_{2}\right) .
$$

Assume with no loss of generality that $\left|\lambda_{1}-k_{1}^{3}\right| \geq\left|\lambda_{2}-k_{2}^{3}\right|$. We will show the first term on the right-side of (2.1) controls the left-side in this case. In the other case, the second term controls the left-side. By defining

$$
\begin{aligned}
& c_{1}\left(k_{1}, \lambda_{1}\right)=\frac{\left(1+\left|k_{1}\right|\right)^{\gamma_{1}-a_{1}}\left|k_{1}\right|^{a_{1}}}{\left(1+\left|\lambda_{1}-k_{1}^{3}\right|\right)^{b}}\left|\widehat{u}\left(k_{1}, \lambda_{1}\right)\right|, \\
& c_{2}\left(k_{2}, \lambda_{2}\right)=\frac{\left(1+\left|k_{2}\right|\right)^{\gamma_{2}-a_{2}}\left|k_{2}\right|^{a_{2}}}{\left(1+\left|\lambda_{2}-k_{2}^{3}\right|\right)^{\beta}}\left|\widehat{v}\left(k_{2}, \lambda_{2}\right)\right|,
\end{aligned}
$$

the desired estimate is renormalized with $L^{2}$ right-side. Indeed, (2.1) follows if we show

$$
\begin{gathered}
\int_{*} \frac{(1+|k|)^{\alpha-a}|k|^{1+a} d(k, \lambda)}{\left(1+\left|\lambda-k^{3}\right|\right)^{1-b}} \frac{\left(1+\left|k_{1}\right|\right)^{-\gamma_{1}+a_{1}}\left|k_{1}\right|^{-a_{1}} c_{1}\left(k_{1}, \lambda_{1}\right)}{\left(1+\left|\lambda_{1}-k_{1}^{3}\right|\right)^{b}} \\
\frac{\left(1+\left|k_{2}\right|\right)^{-\gamma_{2}+a_{2}}\left|k_{2}\right|^{-a_{2}} c_{2}\left(k_{2}, \lambda_{2}\right)}{\left(1+\left|\lambda_{2}-k_{2}^{3}\right|\right)^{\beta}} \\
\leq\|d\|_{L^{2}}\left\|c_{1}\right\|_{L^{2}}\left\|c_{2}\right\|_{L^{2}} .
\end{gathered}
$$


As first exploited by Bourgain [1], we recall the fact that

$$
\max \left\{\left|\lambda-k^{3}\right|,\left|\lambda_{1}-k_{1}^{3}\right|,\left|\lambda_{2}-k_{2}^{3}\right|\right\} \geq C|k|\left|k_{1}\right|\left|k_{2}\right|,
$$

where $k=k_{1}+k_{2}, \lambda=\lambda_{1}+\lambda_{2}$.

We consider Case $\mathbf{A}\left|\lambda-k^{3}\right| \geq\left|\lambda_{1}-k_{1}^{3}\right|$ and then Case B $\left|\lambda-k^{3}\right| \leq\left|\lambda_{1}-k_{1}^{3}\right|$. Because the integral is not symmetric in $k_{1}, k_{2}$, we subdivide Case A into 6 subcases: A.1 $\left|k_{1}\right|,\left|k_{2}\right| \leq 1$, A.2 $\left|k_{1}\right| \leq 1 \ll\left|k_{2}\right|$, A.3 $1 \ll\left|k_{1}\right| \ll\left|k_{2}\right|$, A.4 $1 \ll\left|k_{1}\right| \sim\left|k_{2}\right|$, A.5 $\left|k_{2}\right| \leq 1 \ll\left|k_{1}\right|$, A.6 $1 \ll\left|k_{2}\right| \ll\left|k_{1}\right|$. A similar decomposition is used in Case $\mathrm{B}$.

Before turning to the detailed case-by-case analysis, we record three useful lemmas.

Lemma 1. Assume spt $c_{i} \subset\left\{(k, \lambda):\left|k_{i}\right| \sim M_{i}\right.$ (dyadic) $\}$ for $i=1,2$ and $M_{1}<M_{2}$. Then the following estimate

$$
\int_{*} d(k, \lambda) \frac{c_{1}\left(k_{1}, \lambda_{1}\right)}{\left(1+\left|\lambda_{1}-k_{1}^{3}\right|\right)^{b}} \frac{c_{2}\left(k_{2}, \lambda_{2}\right)}{\left(1+\left|\lambda_{2}-k_{2}^{3}\right|\right)^{b}} \leq C M_{2}^{-1}\|d\|_{L^{2}}\left\|c_{1}\right\|\left\|_{L^{2}}\right\| c_{2} \|_{L^{2}}
$$

holds provided $b>\frac{1}{2}$.

Proof. First, assume that $c_{i}\left(k_{i}, \lambda_{i}\right)=\phi_{i}\left(k_{i}\right) \delta_{\left\{\lambda_{i}=k_{i}^{3}\right\}}$. In this case, the desired estimate is implied by

$$
\begin{gathered}
\int_{\left|k_{i}\right| \sim M_{i}} d\left(k_{1}+k_{2}, k_{1}^{3}+k_{2}^{3}\right) \phi_{1}\left(k_{1}\right) \phi_{2}\left(k_{2}\right) d k_{1} d k_{2} \leq \\
C M_{2}^{-1}\|d\|_{L^{2}}\left\|\phi_{1}\right\|_{L^{2}}\left\|\phi_{2}\right\|_{L^{2}} .
\end{gathered}
$$

To verify (2.5), we change variables $u=k_{1}+k_{2}, v=k_{1}^{3}+k_{2}^{3}$ so that $d u d v=$ $3\left|k_{1}^{2}-k_{2}^{2}\right| d k_{1} d k_{2}$. For $\left|k_{1}\right| \sim M_{1}<M_{2} \sim\left|k_{2}\right|$, we know that $3\left|k_{1}^{2}-k_{2}^{2}\right| \sim M_{2}^{2}$, a fact we will use shortly. The left-side of (2.5) is bounded by

$$
\int d(v, v) H(u, v) d u d v \text { where } H(u, v)=\frac{\left|\phi_{1}\left(k_{1}\right) \phi_{2}\left(k_{2}\right)\right|}{3\left|k_{1}^{2}-k_{2}^{2}\right|} .
$$

We estimate with Cauchy-Schwarz,

$$
\leq\|d\|_{L^{2}}\left(\int H^{2}(u, v) d u d v\right)^{\frac{1}{2}}
$$

and change back to $k_{1}, k_{2}$ to observe that (2.5) holds.

Next, we show the denominators in (2.4) are strong enough to prove the general case using the special case just considered. Decompose $c_{i}$ into cubic level sets by writing

$$
c_{i}\left(k_{i}, \lambda_{i}\right)=\sum_{R_{i} \in \mathbb{Z}} c_{R_{i}}(k, \lambda), \text { spt } c_{R_{i}} \subset\left\{(k, \lambda): \lambda-k^{3}=R_{i}+O(1)\right\} .
$$


The left-side of (2.4) is rewritten

$$
\begin{aligned}
& \sum_{R_{1}, R_{2}} \frac{1}{\left(1+\left|R_{1}\right|\right)^{b}\left(1+\left|R_{2}\right|\right)^{b}} \\
& \int_{\substack{k=k_{1}+k_{2} \\
\lambda=k_{1}^{3}+k_{2}^{3}+R_{1}+R_{2}+\theta_{1}+\theta_{2}}} d(k, \lambda) c_{R_{1}}\left(k_{1}, k_{1}^{3}+R_{1}+\theta_{1}\right) c_{R_{2}}\left(k_{2}, k_{2}^{3}+R_{2}+\theta_{2}\right) .
\end{aligned}
$$

We define $\phi_{R_{i} \theta_{i}}\left(k_{i}\right)=c_{R_{i}}\left(k_{i}, k_{i}^{3}+R_{i}+\theta_{i}\right)$ and rewrite

$$
\begin{aligned}
& \sum_{R_{i}, R_{2}} \frac{1}{\left(1+\left|R_{1}\right|\right)^{b}\left(1+\left|R_{2}\right|\right)^{b}} \\
& \quad \iint d\left(k_{1}+k_{2}, k_{1}^{3}+k_{2}^{3}+R_{1}+R_{2}+\theta_{1}+\theta_{2}\right) \phi_{R_{1} \theta_{1}} \phi_{R_{2} \theta_{2}} d k_{1} d k_{2} d \theta_{1} d \theta_{2} .
\end{aligned}
$$

The $k_{1} k_{2}$-integral is of the form considered in (2.5). Therefore, we can bound by

$$
\sum_{R_{1}, R_{2}} \frac{1}{\left(1+\left|R_{1}\right|\right)^{b}\left(1+\left|R_{2}\right|\right)^{b}} \int M_{2}^{-1}\|d\|_{L^{2}}\left\|\phi_{R_{1} \theta_{1}}\right\|_{L^{2}}\left\|\phi_{R_{2} \theta_{2}}\right\|_{L^{2}} d \theta_{1} d \theta_{2} .
$$

Cauchy-Schwarz in $\theta_{1}, \theta_{2}$ using $\left|\theta_{i}\right| \leq O(1)$ followed by Cauchy-Schwarz in $R_{1}, R_{2}$ using $b>\frac{1}{2}$ proves $(2.4)$.

Lemma 2. Assume spt $c_{i} \subset\left\{(k, \lambda):|k| \sim M_{i}\right\}$ for $i=1,2$ and $M_{1}=M_{2}=$ $M$. Then if $R<<M, \alpha \geq \frac{1}{2}$,

$$
\begin{gathered}
\int_{\substack{k=k_{1}+k_{2} \\
\lambda=\lambda_{1}+\lambda_{2} \\
|k| \leq R}}|k|^{\alpha} \frac{c_{1}\left(k_{1}, \lambda_{1}\right)}{\left(1+\left|\lambda_{1}-k_{1}^{3}\right|\right)^{b}} \frac{c_{2}\left(k_{2}, \lambda_{2}\right)}{\left(1+\left|\lambda_{2}-k_{2}^{3}\right|\right)^{b}} \leq \\
C R^{\alpha-\frac{1}{2}} M^{-\frac{1}{2}}\|d\|_{L^{2}}\left\|c_{1}\right\|_{L^{2}}\left\|c_{2}\right\|_{L^{2}}
\end{gathered}
$$

provided $b>\frac{1}{2}$.

Proof. As in Lemma 1, since $b>\frac{1}{2}$, it suffices to establish this estimate for $c_{i}\left(k_{i}, \lambda_{i}\right)=\phi_{i}\left(k_{i}\right) \delta_{\left\{\lambda_{i}=k_{i}^{3}\right\}}$. So we consider the expression, for arbitrary $\epsilon>0$,

$$
\int_{\substack{\epsilon<\left|k_{1}+k_{2}\right|<R \\\left|k_{i}\right| \sim M}}\left|k_{1}+k_{2}\right|^{\alpha} d\left(k_{1}+k_{2}, k_{1}^{3}+k_{2}^{3}\right) \phi_{1}\left(k_{1}\right) \phi_{2}\left(k_{2}\right) d k_{1} d k_{2} .
$$

Make the change of variable $u=k_{1}+k_{2}, v=k_{1}^{3}+k_{2}^{3}, d u d v=3\left|k_{1}^{2}-k_{2}^{2}\right| d k_{1} d k_{2}$. By noting that $\left|k_{1}^{2}-k_{2}^{2}\right|=\left|k_{1}+k_{2}\right|\left|k_{1}-k_{2}\right|$ and $\left|k_{1}-k_{2}\right| \sim M$, the previous arguments lead us to the desired bound $R^{\alpha-\frac{1}{2}} M^{-\frac{1}{2}}\|d\|_{L^{2}}\left\|c_{1}\right\|_{L^{2}}\left\|c_{2}\right\|_{L^{2}}$ for (2.9).

Similar arguments establish the next result. 
Lemma 3. Assume spt $c_{i} \subset\left\{(k, \lambda):|k| \sim M_{i}\right\}$ for $i=1,2$ and $M_{1}=M_{2}$. Then if $R<<M, \alpha \leq \frac{1}{2}$,

$$
\begin{gathered}
\int_{\substack{k=k_{1}+k_{2} \\
\text { and } \\
|k| \geq R}}|k|^{\alpha} \frac{c_{1}\left(k_{1}, \lambda_{1}\right)}{\left(1+\left|\lambda_{1}-k_{1}^{3}\right|\right)^{b}} \frac{c_{2}\left(k_{2}, \lambda_{2}\right)}{\left(1+\left|\lambda_{2}-k_{2}^{3}\right|\right)^{b}} \leq \\
C R^{\alpha-\frac{1}{2}} M^{-\frac{1}{2}}\|d\|_{L^{2}}\left\|c_{1}\right\|_{L^{2}}\left\|c_{2}\right\|_{L^{2}}
\end{gathered}
$$

provided $b>\frac{1}{2}$.

We return to the case-by-case analysis leading to (2.1).

A.1. $\left|k_{1}\right|,\left|k_{2}\right| \leq 1$. Since $-a \leq 1,-a_{1},-a_{2} \geq 0$, we can estimate the left-side of $(2.2)$ by

$$
\int_{*} \frac{d(k, \lambda)}{\left(1+\left|\lambda-k^{3}\right|\right)^{\mu}} \frac{c_{1}\left(k_{1}, \lambda_{1}\right)}{\left(1+\left|\lambda_{1}-k_{1}^{3}\right|\right)^{b}} \frac{c_{2}\left(k_{2}, \lambda_{2}\right)}{\left(1+\left|\lambda_{2}-k_{2}^{3}\right|\right)^{b}}
$$

where $\mu=1-2 b+\beta$. We have borrowed from the largest denominator to raise the exponent of the other two to $b$. The Strichartz estimate for the cubic [1] implies for $b>\frac{1}{2}$ that

$$
\left\|\int \frac{a(k, \lambda)}{\left(1+\left|\lambda-k^{3}\right|\right)^{b}} e^{i(k x+\lambda t)} d k d \lambda\right\|_{L_{x t}^{8}} \leq C\|a\|_{L^{2}} .
$$

Plancherel's theorem shows the $b=0$ case of $(2.12)$ is valid if $L^{8}$ is replaced by $L^{2}$. By interpolation, the estimate

$$
\left\|\int \frac{a(k, \lambda)}{\left(1+\left|\lambda-k^{3}\right|\right)^{\frac{2}{3}\left(1-\frac{2}{p}\right)+}} e^{i(k x+\lambda t)} d k d \lambda\right\|_{L_{x t}^{p}} \leq C\|a\|_{L^{2}},
$$

holds for $2 \leq p \leq 8$. Hence, we can estimate (2.11),

$$
\|d\|_{L^{2}}\left\|\mathcal{F}^{-1}\left(\frac{c_{1}\left(k_{1}, \lambda_{1}\right)}{\left(1+\left|\lambda_{1}-k_{1}^{3}\right|\right)^{b}}\right)\right\|_{L^{4}}\left\|\mathcal{F}^{-1}\left(\frac{c_{2}\left(k_{2}, \lambda_{2}\right)}{\left(1+\left|\lambda_{2}-k_{2}^{3}\right|\right)^{b}}\right)\right\|_{L^{4}} \leq\|d\|_{L^{2}}\left\|c_{1}\right\|_{L^{2}}\left\|c_{2}\right\|_{L^{2}},
$$

where $\mathcal{F}$ denotes the Fourier transform with respect to $x$ and $t$.

A.2. $\left|k_{1}\right| \leq 1 \ll\left|k_{2}\right| \sim|k|$.

In this region, the quantity to be estimated is

$$
\begin{aligned}
& \int_{*} \frac{|k|^{1+\alpha}\left|k_{1}\right|^{-a_{1}}\left|k_{2}\right|^{-\gamma_{2}}}{\left(1+\left|\lambda-k^{3}\right|\right)^{1-b}\left(1+\left|\lambda_{1}-k_{1}^{3}\right|\right)^{b}\left(1+\left|\lambda_{2}-k_{2}^{3}\right|\right)^{b}} \\
& d(k, \lambda) c_{1}\left(k_{1}, \lambda_{1}\right) c_{2}\left(k_{2}, \lambda_{2}\right) .
\end{aligned}
$$

We consider two subcases: case a $\left|k_{1}\right| \leq \frac{1}{\left|k_{2}\right|^{2}}$, case $\mathbf{b}\left|k_{1}\right| \geq \frac{1}{\left|k_{2}\right|^{2}}$. 
A.2.a. We borrow from the largest denominator and consider

$$
\int_{*}\left|k_{2}\right|^{1+\alpha-\gamma_{2}+2 a_{1}} \frac{d(k, \lambda)}{\left(1+\left|\lambda-k^{3}\right|\right)^{\mu}} \frac{c_{1}\left(k_{1}, \lambda_{1}\right)}{\left(1+\left|\lambda_{1}-k_{1}^{3}\right|\right)^{b}} \frac{c_{2}\left(k_{2}, \lambda_{2}\right)}{\left(1+\left|\lambda_{2}-k_{2}^{3}\right|\right)^{b}} .
$$

Consider the contribution arising form $\left|k_{2}\right| \sim M$, dyadic. Write $c_{M}=c_{2} \chi_{M}$ where $\chi_{M}$ is a cutoff to the region $\left\{\left|k_{2}\right| \sim M\right\}$. The region under consideration here allows us to replace $d$ by $d_{M}=d \chi_{M}$. By Lemma 1, this contribution is estimated

$$
M^{1+\alpha-\gamma_{2}+2 a_{1}} M^{-1}\left\|d_{M}\right\|_{L^{2}}\left\|c_{1}\right\|_{L^{2}}\left\|c_{M}\right\|_{L^{2}} .
$$

Assuming $\alpha-\gamma_{2} \leq-2 a_{1}$, we can sum in dyadic $M$ by applying Cauchy-Schwarz since $\left(\sum_{M}\left\|d_{M}\right\|_{2}^{2}\right)^{\frac{1}{2}}=\|d\|_{L^{2}},\left(\sum_{M}\left\|c_{M}\right\|_{2}^{2}\right)^{\frac{1}{2}}=\|c\|_{L^{2}}$.

Notice that in this case, we have $|k|\left|k_{1}\right|\left|k_{2}\right| \leq C$ so the denominators (2.3) are not useful in controlling derivatives here. Instead, the wide separation of $\left|k_{1}\right|$ and $\left|k_{2}\right|$ allows us to find the factor $\frac{1}{M} \sim \frac{1}{\left|k_{2}\right|}$ by the change of variable argument in the proof of Lemma 1.

A.2.b. $\left|k_{1}\right| \geq\left|k_{2}\right|^{-2} \Longrightarrow(2.3)$ is useful here. We first borrow from the largest denominator to raise $\beta$ to $b$ in (2.14). Then we use (2.3) to control (2.14) by

$$
\int_{*}|k|^{1+\alpha-\mu}\left|k_{2}\right|^{-\gamma_{2}-\mu}\left|k_{1}\right|^{-a_{1}-\mu} d(k, \lambda) \frac{c_{1}\left(k_{1}, \lambda_{1}\right)}{\left(1+\left|\lambda_{1}-k_{1}^{3}\right|\right)^{b}} \frac{c_{2}\left(k_{2}, \lambda_{2}\right)}{\left(1+\left|\lambda_{2}-k_{2}^{3}\right|\right)^{b}} .
$$

If $\mu>-a_{1}$ then we consider the contribution arising from $\left|k_{2}\right| \sim M$, dyadic. This contribution is bounded by

$$
M^{1+\alpha-\mu-\gamma_{2}-\mu+2 a_{1}+2 \mu} \int_{*} d_{M}(k, \lambda) \frac{c_{1}\left(k_{1}, \lambda_{1}\right)}{\left(1+\left|\lambda_{1}-k_{1}^{3}\right|\right)^{b}} \frac{c_{M}\left(k_{2}, \lambda_{2}\right)}{\left(1+\left|\lambda_{2}-k_{2}^{3}\right|\right)^{b}} .
$$

By Lemma 1,

$$
M^{\alpha-\gamma_{2}+2 a_{1}}\left\|d_{M}\right\|_{L^{2}}\left\|c_{1}\right\|_{L^{2}}\left\|c_{2}\right\|_{L^{2}}
$$

and we can sum over dyadic $M \gg 1$ provided $\alpha-\gamma_{2} \leq-2 a_{1}$ by applying Cauchy-Schwarz as before.

If $\mu \leq-a_{1}$, we bound $\left|k_{1}\right|^{-a_{1}-\mu} \leq 1$ and use Lemma 1 to estimate the $\left|k_{2}\right| \sim M$ contribution by

$$
M^{\alpha-\gamma_{2}-2 \mu}\|d\|_{L^{2}}\left\|c_{1}\right\|_{L^{2}}\left\|c_{2}\right\|_{L^{2}}
$$

We can sum provided $\alpha-\gamma_{2}<2 \mu$.

A.3. $1 \ll\left|k_{1}\right| \ll\left|k_{2}\right| \sim|k|$.

The quantity to be estimated here is

$$
\int_{*} \frac{(1+|k|)^{1+\alpha}\left|k_{1}\right|^{-\gamma_{1}}\left|k_{2}\right|^{-\gamma_{2}}}{\left(1+\left|\lambda-k^{3}\right|\right)^{1-b}\left(1+\left|\lambda_{1}-k_{1}{ }^{3}\right|\right)^{b}\left(1+\left|\lambda_{2}-k_{2}{ }^{3}\right|\right)^{\beta}} d(k, \lambda) c_{1}\left(k_{1}, \lambda_{1}\right) c_{2}\left(k_{2}, \lambda_{2}\right) \text {. }
$$


Set $\left|k_{2}\right| \sim|k| \sim M_{2}$ and $\left|k_{1}\right| \sim M_{1}$ for dyadic $M_{1} \ll M_{2}$. Using (2.3), we control this contribution by

$$
M_{2}^{1+\alpha-\gamma_{2}-2 \mu} M_{1}^{-\gamma_{1}-\mu} \int_{*} d(k, \lambda) \frac{c_{1}\left(k_{1}, \lambda_{1}\right)}{\left(1+\left|\lambda_{1}-k_{1}^{3}\right|\right)^{b}} \frac{c_{2}\left(k_{2}, \lambda_{2}\right)}{\left(1+\left|\lambda_{2}-k_{2}^{3}\right|\right)^{b}} .
$$

By Lemma 1 , we control this quantity by

$$
M_{2}^{\alpha-\gamma_{2}-2 \mu} M_{1}^{-\gamma_{1}-\mu}\|d\|_{L^{2}}\left\|c_{1}\right\|_{L^{2}}\left\|c_{2}\right\|_{L^{2}} .
$$

If $-\gamma_{1}-\mu<0$ then we can sum over dyadic $1 \leq M_{1}, M_{2}$ provided $\alpha-\gamma_{2}<$ $2 \mu$. If $-\gamma_{1}-\mu \geq 0$ then, using $1 \ll M_{1} \ll M_{2}$, we can sum if we assume $\alpha-\gamma_{2}-2 \mu-\gamma_{1}-\mu<0$. So we require $\alpha-\gamma_{2}<2 \mu$ and $\alpha-\left(\gamma_{1}+\gamma_{2}\right)<3 \mu$.

A.4. $1 \ll\left|k_{1}\right| \sim\left|k_{2}\right| \sim M$.

We wish to estimate

$$
\begin{aligned}
\int_{*} \frac{|k|^{1+a}(1+|k|)^{\alpha-a}}{\left(1+\left|\lambda-k^{3}\right|\right)^{1-b}} \frac{\left|k_{1}\right|^{-\gamma_{1}}}{\left(1+\left|\lambda_{1}-k_{1}{ }^{3}\right|\right)^{b}} & \frac{\left|k_{2}\right|^{-\gamma_{2}}}{\left(1+\left|\lambda_{2}-k_{2}^{3}\right|\right)^{\beta}} d(k, \lambda) c_{1}\left(k_{1}, \lambda_{1}\right) c_{2}\left(k_{2}, \lambda_{2}\right) .
\end{aligned}
$$

We analyze three subcases: case a $|k|<\frac{1}{M^{2}}$, case $\mathbf{b} \frac{1}{M^{2}} \leq|k|<1$, case c $1 \leq|k|$.

A.4.a. In this case $|k|\left|k_{1}\right|\left|k_{2}\right| \leq C$ so (2.3) is not useful. We borrow from the largest denominator and then use the argument of Kenig, Ponce and Vega [6] to bound (2.15) by

$$
\begin{aligned}
\sup _{k, \lambda} \frac{|k|^{1+a}}{\left(1+\left|\lambda-k^{3}\right|\right)^{\mu}} & \\
& \left(\int_{*} \frac{\left|k_{1}\right|^{-\gamma_{1}}\left|k_{2}\right|^{-\gamma_{2}}}{\left(1+\left|\lambda_{1}-k_{1}^{3}\right|\right)^{2 b}\left(1+\left|\lambda_{2}-k_{2}^{3}\right|\right)^{2 b}}\right)^{\frac{1}{2}}\|d\|_{L^{2}}\left\|c_{1}\right\|_{L^{2}}\left\|c_{2}\right\|_{L^{2}} .
\end{aligned}
$$

The calculus lemmas in [6] show the prefactor above is bounded by

$$
\frac{|k|^{\frac{3}{4}+a} M^{-\left(\gamma_{1}+\gamma_{2}\right)}}{\left(1+\left|\lambda-k^{3}\right|\right)^{\mu}} \frac{1}{\left(1+\left|\lambda-\frac{1}{4} k^{3}\right|\right)^{\frac{1}{4}}} .
$$

We ignore the denominators in this expression and, noting that $\frac{3}{4}+a>0$, use $|k|<\frac{1}{M^{2}}$ to observe this contribution is bounded by $M^{-\frac{3}{2}-2 a-\left(\gamma_{1}+\gamma_{2}\right)}$. This sums over dyadic $M$ provided $-\left(\gamma_{1}+\gamma_{2}\right)<\frac{3}{2}+2 a$. 
A.4.b. Since $\frac{1}{M^{2}} \leq|k| \leq 1,(2.3)$ is useful in this region. We bound (2.15) by

$$
M^{-\left(\gamma_{1}+\gamma_{2}\right)-2 \mu} \int_{*}\left|k_{1}+k_{2}\right|^{a+1-\mu} d(k, \lambda) \frac{c_{1}\left(k_{1}, \lambda_{1}\right)}{\left(1+\left|\lambda_{1}-k_{1}^{3}\right|\right)^{b}} \frac{c_{2}\left(k_{2}, \lambda_{2}\right)}{\left(1+\left|\lambda_{2}-k_{2}^{3}\right|\right)^{b}} .
$$

If $a>\mu-\frac{1}{2}$ then by Lemma 2 we bound by

$$
M^{-\left(\gamma_{1}+\gamma_{2}\right)-2 \mu-\frac{1}{2}}\|d\|_{L^{2}}\left\|c_{1}\right\|_{L^{2}}\left\|c_{2}\right\|_{L^{2}} .
$$

So we require $-\left(\gamma_{1}+\gamma_{2}\right)<2 \mu+\frac{1}{2}$. If $a \leq \mu-\frac{1}{2}$ we use Lemma 3 to bound by

$$
M^{-\left(\gamma_{1}+\gamma_{2}\right)-2 \mu-\frac{1}{2}}\left(\frac{1}{M^{2}}\right)^{a+\frac{1}{2}-\mu}\|d\|_{L^{2}}\left\|c_{1}\right\|_{L^{2}}\left\|c_{2}\right\|_{L^{2}} .
$$

So, we require $-\left(\gamma_{1}+\gamma_{2}\right)<2 a+\frac{3}{2}$.

A.4.c. $\left|k_{1}+k_{2}\right| \geq 1$.

Here we wish to bound

$$
\int_{*} \frac{|k|^{1+\alpha}\left|k_{1}\right|^{-\gamma_{1}}\left|k_{2}\right|^{-\gamma_{2}}}{\left(1+\left|\lambda-k^{3}\right|\right)^{\mu}\left(1+\left|\lambda_{1}-k_{1}{ }^{3}\right|\right)^{b}\left(1+\left|\lambda_{2}-k_{2}{ }^{3}\right|\right)^{b}} d(k, \lambda) c_{1}\left(k_{1}, \lambda_{1}\right) c_{2}\left(k_{2}, \lambda_{2}\right) .
$$

Again, by the argument of [6], it suffices to show

$$
\sup _{k, \lambda} \frac{|k|^{1+\alpha}}{\left(1+\left|\lambda-k^{3}\right|\right)^{\mu}}\left(\int_{*} \frac{\left|k_{1}\right|^{-2 \gamma_{1}}\left|k_{2}\right|^{-2 \gamma_{2}}}{\left(1+\left|\lambda_{1}-k_{1}{ }^{3}\right|\right)^{2 b}\left(1+\left|\lambda_{2}-k_{2}{ }^{3}\right|\right)^{2 b}}\right)^{\frac{1}{2}} \leq C .
$$

Recall that $|k|\left|k_{1}\right|\left|k_{2}\right| \leq C\left|\lambda-k^{3}\right|$. So we rearrange the numerator by writing

$$
\begin{aligned}
\left|k_{1}\right|^{-2 \gamma_{1}}\left|k_{2}\right|^{-2 \gamma_{2}} \sim\left(\left|k_{1}\right|\left|k_{2}\right|\right)^{-\left(\gamma_{1}+\gamma_{2}\right)} & \sim|k|^{\left(\gamma_{1}+\gamma_{2}\right)}\left(|k|\left|k_{1}\right|\left|k_{2}\right|\right)^{-\left(\gamma_{1}+\gamma_{2}\right)} \\
& \leq C|k|^{\left(\gamma_{1}+\gamma_{2}\right)}\left(1+\left|\lambda-k^{3}\right|\right)^{-\left(\gamma_{1}+\gamma_{2}\right)} .
\end{aligned}
$$

So, (2.16) is controlled by

$$
\begin{aligned}
\sup _{k, \lambda}|k|^{1+\alpha+\frac{\left(\gamma_{1}+\gamma_{2}\right)}{2}}\left(1+\left|\lambda-k^{3}\right|\right)^{-\left(\frac{\gamma_{1}+\gamma_{2}}{2}\right)-\mu} & \\
& \left(\int_{*} \frac{d k_{1} d \lambda_{1}}{\left(1+\left|\lambda_{1}-k_{1}{ }^{3}\right|\right)^{2 b}\left(1+\left|\lambda_{2}-k_{2}{ }^{3}\right|\right)^{2 b}}\right)^{\frac{1}{2}} .
\end{aligned}
$$

Estimating the integral as in [6], we reduce matters to showing

$$
\sup _{k, \lambda} \frac{|k|^{1+\alpha+\left(\frac{\gamma_{1}+\gamma_{2}}{2}\right)-\frac{1}{4}}\left(1+\left|\lambda-k^{3}\right|\right)^{-\left(\frac{\gamma_{1}+\gamma_{2}}{2}\right)-\mu}}{\left(1+\left|\lambda-\frac{1}{4} k^{3}\right|\right)^{\frac{1}{4}}} \leq C .
$$

Now we consider three cases depending on the relative size of $|\lambda|$ and $|k|^{3}$.

A.4.c.i. $|\lambda| \leq \frac{1}{8}|k|^{3} \Longrightarrow\left|\lambda-k^{3}\right| \sim\left|\lambda-\frac{1}{4} k^{3}\right| \sim|k|^{3}$. Then boundedness and summability in $M$ is implied by $1+\alpha+\left(\frac{\gamma_{1}+\gamma_{2}}{2}\right)-\frac{1}{4}-3\left(\frac{\gamma_{1}+\gamma_{2}}{2}\right)-3 \mu-\frac{3}{4}<0$ which unravels to $\alpha-\left(\gamma_{1}+\gamma_{2}\right)<3 \mu$. 
A.4.c.ii. $\frac{1}{8}|k|^{3} \leq|\lambda| \leq \frac{1}{2}|k|^{3} \Longrightarrow\left|\lambda-k^{3}\right| \sim|k|^{3}$. For boundedness, we ask that $\alpha-\left(\gamma_{1}+\gamma_{2}\right)<3 \mu-\frac{3}{4}$.

A.4.c.iii. $\frac{1}{2}|k|^{3} \leq|\lambda| \Longrightarrow\left|\lambda-\frac{1}{4} k^{3}\right| \sim|\lambda| \gtrsim|k|^{3}$. This case seems to allow $\left|\lambda-k^{3}\right|=0$. However, since we are in case A.4.c, we know $\left|\lambda-k^{3}\right| \geq C|k|\left|k_{1}\right|\left|k_{2}\right|$ and $\left|k_{1}\right| \sim\left|k_{2}\right| \sim M \gg 1$ and $|k| \geq 1$. Since $\left|\lambda-\frac{1}{4} k^{3}\right| \geq C\left|\lambda-k^{3}\right|$ in this region we can bound (2.17) by

$$
|k|^{\frac{3}{4}+\alpha+\left(\frac{\gamma_{1}+\gamma_{2}}{2}\right)}\left|\lambda-k^{3}\right|^{-\left(\frac{\gamma_{1}+\gamma_{2}}{2}\right)-\mu-\frac{1}{4}} .
$$

We require that the exponent on $\left|\lambda-k^{3}\right|$ be negative. Then, using $\left|\lambda-k^{3}\right| \geq$ $C|k|\left|k_{1}\right|\left|k_{2}\right| \geq C|k|^{3}$ we have the desired boundedness and summability if $\alpha-$ $\left(\gamma_{1}+\gamma_{2}\right)<3 \mu$.

A.5. $\left|k_{2}\right| \leq 1 \ll\left|k_{1}\right| \sim|k| \sim M$.

By borrowing from the largest denominator, this contribution may be estimated

$$
M^{1+\alpha-\gamma_{1}} \int_{*}\left|k_{2}\right|^{-a_{2}} \frac{d(k, \lambda)}{\left(1+\left|\lambda-k^{3}\right|\right)^{\mu}} \frac{c_{1}\left(k_{1}, \lambda_{1}\right)}{\left(1+\left|\lambda_{1}-k_{1}^{3}\right|\right)^{b}} \frac{c_{2}\left(k_{2}, \lambda_{2}\right)}{\left(1+\left|\lambda_{2}-k_{2}^{3}\right|\right)^{b}} .
$$

This is treated like Case A.2. We obtain the following conditions: If $\mu \geq-a_{2}$, we require $\alpha-\gamma_{1}<-2 a_{2}$ and if $\mu \leq-a_{2}$, we require $\alpha-\gamma_{1}<2 \mu$.

A.6. $1 \ll\left|k_{2}\right| \ll\left|k_{1}\right|$. This is like Case A.3 and gives the condition $\alpha-\gamma_{2}<2 \mu$ and $\alpha-\left(\gamma_{1}+\gamma_{2}\right)<3 \mu$. This ends the discussion of Case A.

Case B. $\left|\lambda_{1}-k_{1}^{3}\right| \geq\left|\lambda-k^{3}\right|$.

We wish to prove (2.2). By a simple change of variable, we can rewrite the left-side as

$$
\begin{array}{r}
\int_{*} \frac{(1+|k|)^{-\gamma_{1}+a_{1}}|k|^{-a_{1}}\left(1+\left|k_{1}\right|\right)^{\alpha-a}\left|k_{1}\right|^{1+a}\left(1+\left|k_{2}\right|\right)^{\gamma_{2}-a_{2}}\left|k_{2}\right|^{-a_{2}}}{\left(1+\left|\lambda-k^{3}\right|\right)^{b}\left(1+\left|\lambda_{1}-k_{1}^{3}\right|\right)^{1-b}\left(1+\left|\lambda_{2}-k_{2}{ }^{3}\right|\right)^{\beta}} \\
c_{1}(k, \lambda) d^{-}\left(k_{1}, \lambda_{1}\right) c_{2}^{-}\left(k_{2}, \lambda_{2}\right)
\end{array}
$$

where $d^{-}(k, \lambda)=d(-k,-\lambda)$. We distinguish the following cases: B.1 $\left|k_{2}\right|,\left|k_{1}\right| \leq$ 1 , B.2 $\left|k_{2}\right| \leq 1 \ll\left|k_{1}\right|$, B.3 $1 \ll\left|k_{2}\right| \ll\left|k_{1}\right|$, B.4 $1 \ll\left|k_{2}\right| \sim\left|k_{1}\right|$, B.5 $\left|k_{1}\right| \leq 1 \ll\left|k_{2}\right|$, B.6 $1 \ll\left|k_{1}\right| \ll\left|k_{2}\right|$. Note that in these new variables, we now have $\left|\lambda-k^{3}\right|=\max \left(\left|\lambda-k^{3}\right|,\left|\lambda_{1}-k_{1}^{3}\right|,\left|\lambda_{2}-k_{2}^{3}\right|\right)$.

B.1. This is the same as Case A.1 discussed above after we redistribute the largest denominator.

B.2. $\left|k_{2}\right| \leq 1 \ll\left|k_{1}\right|$. 
B.2.a. $\left|k_{2}\right|<\frac{1}{M^{2}}$. We bound (2.19) in this region by

$$
M^{-\gamma_{1}+\alpha+1+2 a_{2}} \int_{*} c_{M}(k, \lambda) \frac{c_{2}\left(k_{2}, \lambda_{2}\right)}{\left(1+\left|\lambda_{2}-k_{2}^{3}\right|\right)^{b}} \frac{d_{M}\left(k_{1}, \lambda_{1}\right)}{\left(1+\left|\lambda_{1}-k_{1}^{3}\right|\right)^{b}}
$$

where $c_{M}=c_{1} \chi_{M}, d_{M}=d \chi_{M}$ and $\chi_{M}$ is a cutoff to $\{|k| \sim M\}$. By lemma 1, this is bounded by

$$
M^{-\gamma_{1}+\alpha+2 a_{2}}\left\|c_{M}\right\|_{L^{2}}\left\|c_{2}\right\|_{L^{2}}\left\|d_{M}\right\|_{L^{2}}
$$

We can sum over dyadic $M$ provided $\alpha-\gamma_{1} \leq-2 a_{2}$.

B.2.b. $\frac{1}{M^{2}} \leq\left|k_{2}\right|$. This is similar to Case A.2.b considered above. In case $\mu>-a_{2}$ we require $\alpha-\gamma_{1} \leq-2 a_{2}$. If $\mu \leq-a_{2}$, we require $\alpha-\gamma_{1}<2 \mu$.

B.3. $1 \ll\left|k_{2}\right| \ll\left|k_{1}\right| \sim|k|$. This can be treated like Case A.3 above provided $\alpha-\gamma_{1}<2 \mu$ and $\alpha-\left(\gamma_{1}+\gamma_{2}\right)<3 \mu$.

B.4. $1 \ll\left|k_{1}\right| \sim\left|k_{2}\right| \sim M$. We subdivide into two cases: case a $\left|k_{1}+k_{2}\right| \leq 1$, case $\mathbf{b}\left|k_{1}+k_{2}\right| \geq 1$.

B.4.a. We wish to bound

$$
\int_{*} \frac{|k|^{-a_{1}}\left|k_{1}\right|^{\alpha+1}\left|k_{2}\right|^{-\gamma_{2}}}{\left(1+\left|\lambda-k^{3}\right|\right)^{\mu}\left(1+\left|\lambda_{1}-k_{1}{ }^{3}\right|\right)^{b}\left(1+\left|\lambda_{2}-k_{2}{ }^{3}\right|\right)^{b}} c(k, \lambda) \tilde{c}_{M}\left(k_{2}, \lambda_{2}\right) d_{M}\left(k_{1}, \lambda_{1}\right)
$$

where $d_{M}=d \chi_{M}$ and $\tilde{c}_{M}=c_{2} \chi_{M}$.

B.4.a.i. $\left|k_{1}+k_{2}\right| \leq \frac{1}{M^{\rho}}(\rho>0$ is selected below $)$. We borrow from the largest denominator and bound (2.20) in this region by

$$
M^{\rho a_{1}} M^{\alpha+1-\gamma_{2}} \int_{*} \frac{c(k, \lambda)}{\left(1+\left|\lambda-k^{3}\right|\right)^{b}} d\left(k_{1}, \lambda_{1}\right) \frac{\tilde{c}_{M}\left(k_{2}, \lambda_{2}\right)}{\left(1+\left|\lambda_{1}-k_{1}^{3}\right|\right)^{b}} .
$$

Since $c$ is supported in $\{|k| \ll M\}$ and $\tilde{c}_{M}$ is supported in $\left\{\left|k_{2}\right| \sim M\right\}$, we have a wide frequency separation and can apply Lemma 1 . We obtain

$$
M^{\rho a_{1}} M^{\alpha-\gamma_{2}}\|c\|_{L^{2}}\left\|\tilde{c}_{M}\right\|_{L^{2}}\left\|d_{M}\right\|_{L^{2}} .
$$

We will require that $\alpha-\gamma_{2} \leq-\rho a_{1}$. Then, Cauchy-Schwarz in $M$ establishes the desired estimate here. 
B.4.a.ii. $\frac{1}{M^{\rho}} \leq\left|k_{1}+k_{2}\right| \leq 1$. Provided we select $0<\rho<2$, this condition makes (2.3) useful in reducing the powers of $|k|$ and $\left|k_{i}\right|$ in the numerator. Upon borrowing from the largest denominator and using (2.3), we bound (2.19) by

$$
\begin{aligned}
& \leq \int_{*} \frac{|k|^{-a_{1}-\mu}\left|k_{1}\right|^{\alpha+1-\gamma_{2}-2 \mu}}{\left(1+\left|\lambda_{1}-k_{1}^{3}\right|\right)^{b}\left(1+\left|\lambda_{2}-k_{2}{ }^{3}\right|\right)^{b}} c(k, \lambda) \tilde{c}_{M}\left(k_{2}, \lambda_{2}\right) d_{M}\left(k_{1}, \lambda_{1}\right) \\
& \leq M^{\alpha+1-\gamma_{2}-2 \mu} \int_{*}|k|^{-a_{1}-\mu} c_{M}(k, \lambda) \frac{d_{M}\left(k_{1}, \lambda_{1}\right)}{\left(1+\left|\lambda_{1}-k_{1}{ }^{3}\right|\right)^{b}} \frac{\tilde{c}_{M}\left(k_{2}, \lambda_{2}\right)}{\left(1+\left|\lambda_{2}-k_{2}{ }^{3}\right|\right)^{b}} .
\end{aligned}
$$

Since $-a_{1}-\mu<\frac{1}{2}$, we estimate using Lemma 3 to get

$$
M^{\alpha+1-\gamma_{2}-2 \mu-\frac{1}{2}} M^{\rho\left(a_{1}+\mu+\frac{1}{2}\right)}\left\|c_{M}\right\|_{L^{2}}\left\|\tilde{c}_{M}\right\|_{L^{2}}\|d\|_{L^{2}} .
$$

We want $\rho\left(a_{1}+\mu+\frac{1}{2}\right)+\alpha-\gamma_{2}+\frac{1}{2}-2 \mu \leq 0$ so we can sum over dyadic $M$. If we set $\rho=\frac{2 \mu-\frac{1}{2}}{\mu+\frac{1}{2}} \in\left[0, \frac{1}{2}\right]$ for $\mu \in\left[\frac{1}{4}, \frac{1}{2}\right]$ then we require $\alpha-\gamma_{2} \leq\left(-a_{2}\right)\left(\frac{2 \mu-\frac{1}{2}}{\mu+\frac{1}{2}}\right)$.

B.4.b. $1 \leq\left|k_{1}+k_{2}\right|,\left|k_{1}\right| \sim\left|k_{2}\right|$. The argument of [6] reduces matters to showing that

$$
\sup _{k \lambda} \frac{1}{\left(1+\left|\lambda-k^{3}\right|\right)^{\mu}}\left(\int_{*} \frac{|k|^{-2 \gamma_{1}}\left|k_{1}\right|^{2 \alpha+2}\left|k_{2}\right|^{-2 \gamma_{2}}}{\left(1+\left|\lambda_{1}-k_{1}{ }^{3}\right|\right)^{2 b}\left(1+\left|\lambda_{2}-k_{2}{ }^{3}\right|\right)^{2 b}}\right)^{\frac{1}{2}} \leq C
$$

As in Case A.4 above, we reorganize the numerator by writing $\left|k_{1}\right|^{2 \alpha+2}|k|^{-2 \gamma_{1}}\left|k_{2}\right|^{-2 \gamma_{2}}=\left(|k|\left|k_{1}\right|\left|k_{2}\right|\right)^{1+\alpha-\gamma_{2}}|k|^{-2 \gamma_{1}-\left(1+\alpha-\gamma_{2}\right)}$ using $\left|k_{1}\right| \sim\left|k_{2}\right|$. Because $\alpha-\gamma_{2}>-1$ and $\left|\lambda-k^{3}\right|>c|k|\left|k_{1}\right|\left|k_{2}\right|$ we bound the square of (2.21) by

$$
\begin{aligned}
\sup _{k \lambda}\left(1+\left|\lambda-k^{3}\right|\right)^{1+\alpha-\gamma_{2}-2 \mu}|k|^{-2 \gamma_{1}-\alpha+\gamma_{2}-1} & \\
& \int_{*} \frac{1}{\left(1+\left|\lambda_{1}-k_{1}^{3}\right|\right)^{2 b}\left(1+\left|\lambda_{2}-k_{2}{ }^{3}\right|\right)^{2 b}} .
\end{aligned}
$$

The change of variables argument from [6] gives the bound

$$
\frac{\left(1+\left|\lambda-k^{3}\right|\right)^{1+\alpha-\gamma_{2}-2 \mu}|k|^{-2 \gamma_{1}-\alpha+\gamma_{2}-1-\frac{1}{2}}}{\left(1+\left|\lambda-\frac{1}{4} k^{3}\right|\right)^{\frac{1}{2}}} .
$$

An analysis of this expression in the three regions $|\lambda|<\frac{1}{8}\left|k^{3}\right|, \quad \frac{1}{8}\left|k^{3}\right| \leq|\lambda| \leq$ $\frac{1}{2}\left|k^{3}\right|, \quad \frac{1}{2}\left|k^{3}\right|<|\lambda|$ as in Case A.4 leads to the parameter restrictions $\alpha-\left(\gamma_{1}+\right.$ $\left.\gamma_{2}\right)<3 \mu, \alpha-\left(\gamma_{1}+\gamma_{2}\right)<3 \mu-\frac{3}{4}, \alpha-\gamma_{2}<2 \mu-\frac{1}{2}$.

B.5. $\left|k_{1}\right| \leq 1 \ll\left|k_{2}\right| \sim|k| \sim M$. 
B.5.a. $\left|k_{1}\right|<\frac{1}{M^{2}}$.

$$
M^{-\left(\gamma_{1}+\gamma_{2}\right)} \int_{*}\left|k_{1}\right|^{1+a} \frac{c_{1}(k, \lambda)}{\left(1+\left|\lambda-k^{3}\right|\right)^{\mu}} \frac{d\left(k_{1}, \lambda_{1}\right)}{\left(1+\left|\lambda_{1}-k_{1}^{3}\right|\right)^{b}} \frac{c_{2}\left(k_{2}, \lambda_{2}\right)}{\left(1+\left|\lambda_{2}-k_{2}^{3}\right|\right)^{b}} .
$$

Apply Lemma 1 to bound by

$$
M^{-\left(\gamma_{1}+\gamma_{2}\right)-2-2 a-1}\left\|c_{1}\right\|_{L^{2}}\|d\|_{L^{2}}\left\|c_{2}\right\|_{L^{2}} .
$$

To sum in dyadic $M$, we require $-\left(\gamma_{1}+\gamma_{2}\right)<3+2 a$.

B.5.b. $\frac{1}{M^{2}} \leq\left|k_{1}\right| \leq 1$. This condition ensures that (2.3) is useful. So we bound by

$$
M^{-\left(\gamma_{1}+\gamma_{2}\right)-2 \mu} \int_{*}\left|k_{1}\right|^{a+1-\mu} c_{1}(k, \lambda) \frac{d\left(k_{1}, \lambda_{1}\right)}{\left(1+\left|\lambda_{1}-k_{1}^{3}\right|\right)^{b}} \frac{c_{2}\left(k_{2}, \lambda_{2}\right)}{\left(1+\left|\lambda_{2}-k_{2}^{3}\right|\right)^{b}} .
$$

Since $a+1-\mu \geq 0$, we can use $\left|k_{1}\right|^{a+1-\mu} \leq 1$ and Lemma 1 to obtain

$$
M^{-\left(\gamma_{1}+\gamma_{2}\right)-2 \mu-1}\|d\|_{L^{2}}\left\|c_{1}\right\|_{L^{2}}\left\|c_{2}\right\|_{L^{2}} .
$$

To sum in dyadic $M$, we require $-\left(\gamma_{1}+\gamma_{2}\right)<1+2 \mu$.

B.6. $1 \ll\left|k_{1}\right| \ll\left|k_{2}\right| \sim|k| \sim M$. This is similar to Case A.4.c. We bound (2.19) by

$$
\int_{*} \frac{|k|^{-\gamma_{1}}\left|k_{1}\right|^{1+\alpha}\left|k_{2}\right|^{-\gamma_{2}}}{\left(1+\left|\lambda-k^{3}\right|\right)^{\mu}\left(1+\left|\lambda_{1}-k_{1}{ }^{3}\right|\right)^{b}\left(1+\left|\lambda_{2}-k_{2}{ }^{3}\right|\right)^{b}} c_{1}(k, \lambda) d\left(k_{1}, \lambda_{1}\right) c_{2}\left(k_{2}, \lambda_{2}\right) .
$$

Since (2.3) is useful in this region, we write

$$
M^{-\left(\gamma_{1}+\gamma_{2}\right)-2 \mu} \int_{*}\left|k_{1}\right|^{1+\alpha-\mu} c_{1}(k, \lambda) \frac{d\left(k_{1}, \lambda_{1}\right)}{\left(1+\left|\lambda_{1}-k_{1}^{3}\right|\right)^{b}} \frac{c_{2}\left(k_{2}, \lambda_{2}\right)}{\left(1+\left|\lambda_{2}-k_{2}^{3}\right|\right)^{b}} .
$$

If $1+\alpha-\mu \geq 0$, we can use Lemma 1 to bound by

$$
M^{-\left(\gamma_{1}+\gamma_{2}\right)-2 \mu+1+\alpha-\mu} M^{-1}\left\|c_{1}\right\|_{L^{2}}\|d\|_{L^{2}}\left\|c_{2}\right\|_{L^{2}} .
$$

We require $\alpha-\left(\gamma_{1}+\gamma_{2}\right)<3 \mu$. If instead, we have $1+\alpha-\mu<0$ then $\left|k_{1}\right|^{1+\alpha-\mu} \leq 1$ and Lemma 1 leads to the condition $-\left(\gamma_{1}+\gamma_{2}\right)<2 \mu+1$.

We conclude this section by showing the necessity of some of the parameter restrictions in the statement of Theorem 2. In case $\mu=b$ and $a=a_{1}=a_{2}=0$ with $\alpha=\gamma_{1}=\gamma_{2}$, the counterexample in [6] shows $\alpha=\gamma_{1}=\gamma_{2}>-\frac{3}{4}$ is necessary for the bilinear estimate to hold. The need for the homogeneous weights of negative index is demonstrated by the following statement.

Proposition 1. For any $b \in \mathbb{R}$, the bilinear estimate

$$
\left\|\partial_{x}(u v)\right\|_{X_{0,0, b-1}} \leq C\|u\|_{X_{\gamma, 0, b}}\|v\|_{X_{\gamma, 0, b}}
$$

fails for $\gamma<0$. 
Proof. Fix $N \gg 1$. Let $R_{(k, \lambda)}^{N}$ be a thin rectangle of side $N^{-2}$ in the $k$-direction and side 1 in the $\lambda$-direction centered at the point $(k, \lambda)$. Set

$$
\widehat{u}(k, \lambda)=\chi_{R_{\left(N, N^{3}\right)}^{N}}(k, \lambda), \widehat{v}(k, \lambda)=\chi_{R_{(0,0)}^{N}}(k, \lambda) .
$$

Then

$$
(\widehat{u} * \widehat{v})(k, \lambda) \sim\left|R^{N}\right| \chi_{\tilde{R}_{\left(N, N^{3}\right)}^{N}} \sim N^{-2} \chi_{\tilde{R}_{\left(N, N^{3}\right)}^{N}},
$$

where $\tilde{R}$ denotes the double of $R$. We calculate the left-side of (2.22),

$$
\begin{aligned}
\left\|\partial_{x}(u v)\right\|_{X_{0,0, b-1}} & \sim\left(\iint \frac{|k|^{2}}{\left(1+\left|\lambda-k^{3}\right|\right)^{2(1-b)}}\left|N^{-2} \chi_{\tilde{R}_{\left(N, N^{3}\right)}^{N}}(k, \lambda)\right|^{2} d k d \lambda\right)^{\frac{1}{2}} \\
& \sim N N^{-2}\left(N^{-2}\right)^{\frac{1}{2}} .
\end{aligned}
$$

The right-side of $(2.22)$ is calculated

$$
\|u\|_{X_{\gamma, 0, \frac{1}{2}}} \sim N^{\gamma}\left(N^{-2}\right)^{\frac{1}{2}},\|v\|_{X_{\gamma, 0, \frac{1}{2}}} \sim\left(N^{-2}\right)^{\frac{1}{2}} .
$$

For the estimate (2.22) to hold, we must have $C \leq N^{\gamma}$ for all $N \gg 1$ which fails for $\gamma<0$.

\section{Local wellposedness}

We begin by considering the initial value problem

$$
\left\{\begin{array}{c}
\partial_{t} u+\partial_{x}^{3} u+\frac{1}{2} \partial_{x} u^{2}=0 \\
u(0)=\phi_{0} \in H^{0, a}(\mathbb{R}), \text { for certain } a \leq 0
\end{array}\right.
$$

Since $H^{0, a} \subset L^{2}$, Bourgain's result [1] shows this problem is locally wellposed. We revisit Bourgain's argument and record the size of the existence interval in terms of the $L^{2}$ norm of the initial data. Moreover, we show the solution $u(t)$ remains in $H^{0, a}$.

Next, we consider the initial value problem

$$
\left\{\begin{array}{c}
\partial_{t} v+\partial_{x}^{3} v+\frac{1}{2} \partial_{x}\left(v^{2}+2 u(x, t) v\right)=0 \\
v(0)=\psi_{0} \in H^{\sigma, \tilde{a}}(\mathbb{R}), \sigma, \tilde{a}<0
\end{array}\right.
$$

where the variable coefficient $u \in X_{0, a, b}$, and $\left\|\psi_{0}\right\|_{H^{\sigma, \tilde{a}}} \ll 1$. We establish local wellposedness of (3.2) and show the size of the existence interval is determined by $\|u\|_{X_{0,0, b}^{\delta}}$ provided $\left\|\psi_{0}\right\|_{H^{\sigma, \tilde{a}}}$ is small enough. When we take the variable coefficient $u$ to be the solution of (3.1), the wellposedness result of (3.1) permits us to show the existence interval for (3.2) is determined by $\left\|\phi_{0}\right\|_{L^{2}}$.

Proposition 2. The initial value problem (3.1) is wellposed on a time interval $[0, \delta]$ where $\delta$ satisfies

$$
\delta^{\frac{1}{4}-}\left\|\phi_{0}\right\|_{L^{2}} \sim 1 .
$$


Moreover, the solution u satisfies

$$
\left\|u_{0}\right\|_{X_{0, a, b}^{\delta}} \leq C\left\|\phi_{0}\right\|_{H^{0, a}}, \quad b=\frac{1}{2}+.
$$

Proof. The problem (3.1) is equivalent to finding $u$ which satisfies

$$
u(t)=S(t) \phi_{0}-\int_{0}^{t} S(t-\tau) \frac{1}{2} \partial_{x} u^{2}(\tau) d \tau .
$$

Denote the right-side of $(3.5)$ by $\Phi_{\phi_{0}}(u)$. We will show $\Phi_{\phi_{0}}$ is a contraction on $X_{0, a, b}^{\delta}$ with $b=\frac{1}{2}+$ for appropriate $-\frac{3}{4}<a<0, \delta>0$ selected below.

Step 1. $\Phi_{\phi_{0}}$ : Bounded subsets of $X_{0, a, b}^{\delta} \longmapsto$ Bounded subsets of $X_{0, a, b}^{\delta}$. We have for $b=\frac{1}{2}+$ and $\epsilon_{b}=b-\frac{1}{2}=0+$,

$$
\begin{aligned}
\left\|S(t) \phi_{0}\right\|_{X_{0, a, b}^{\delta}} & \leq C \delta^{-\epsilon_{b}}\left\|\phi_{0}\right\|_{H^{0, a}}, a \in \mathbb{R}, \\
\left\|\int_{0}^{t} S(t-\tau) \partial_{x} u^{2}(\tau) d \tau\right\|_{X_{0, a, b}^{\delta}} & \leq C \delta^{-\epsilon_{b}}\left\|\partial_{x} u^{2}\right\|_{X_{0, a, b-1}^{\delta}},
\end{aligned}
$$

by adapting the proofs of Lemmas 3.1 and 3.3 in [6]. The bilinear estimate implies

$$
\left\|\partial_{x} u^{2}\right\|_{X_{0, a, b-1}} \leq C\|u\|_{X_{0, a, b}}\|u\|_{X_{0, a, \beta}}
$$

provided $b=\frac{1}{2}+, \frac{1}{4}<\beta<\frac{1}{2}$ and $-\frac{3}{4}<a \leq 0$. Since $X_{0, a, \beta}^{\delta} \subset X_{0, a, b}^{\delta}$, we have that bounded subsets in $X_{0, a, b}^{\delta}$ map into bounded subsets in $X_{0, a, b}^{\delta}$ under the mapping $\Phi_{\phi_{0}}$.

Step 2. Shrink the constant for contraction.

Allowing $\beta<\frac{1}{2}$ permits us to exploit the size of the time interval in the definition of $X_{s, b}^{\delta}$. By interpolation,

$$
\|u\|_{X_{\gamma, a, \beta}^{\delta}} \leq\|u\|_{X_{\gamma, a, 0}^{\delta}}^{1-\frac{\beta}{b}}\|u\|_{X_{\gamma, a, b}^{\delta}}^{\frac{\beta}{b}} .
$$

It can be shown that

$$
\|u\|_{X_{\gamma, a, 0}^{\delta}} \leq C \delta^{\frac{1}{2}}\|u\|_{X_{\gamma, a, b}^{\delta}}
$$

Indeed, we have

$$
\|u\|_{X_{\gamma, a, 0}^{\delta}}^{2}=\int\left[\chi_{\{|k| \geq 1\}}(k)(1+|k|)^{2 \gamma}+\chi_{\{|k|<1\}}(k)|k|^{2 a}\right]\|\widehat{u}(k)(\cdot)\|_{L_{\delta}^{2}} d k,
$$

where $\widehat{u}$ here refers to the Fourier transform with respect to the spatial variable $x$. Since $u$ is supported in a $\delta$-sized interval in the $t$-variable, we have

$$
\leq \int\left[\chi_{\{|k| \geq 1\}}(k)(1+|k|)^{2 \gamma}+\chi_{\{|k|<1\}}(k)|k|^{2 a}\right] \delta\|\widehat{u}(k)(\cdot)\|_{L_{\delta}^{\infty}}^{2} d k .
$$


Finally, we take the Fourier transform with respect to $t$ and use Cauchy-Schwarz with $b>\frac{1}{2}$ to get

$$
\begin{aligned}
& \leq \int\left[\chi_{\{|k| \geq 1\}}(k)(1+|k|)^{2 \gamma}+\chi_{\{|k|<1\}}(k)|k|^{2 a}\right] \delta\left(\int|\widehat{u}(k, \lambda)| d \lambda\right)^{2} d k \\
& \leq \delta \int\left[\chi_{\{|k| \geq 1\}}(k)(1+|k|)^{2 \gamma}+\chi_{\{|k|<1\}}(k)|k|^{2 a}\right] \\
& \qquad\left(\int \frac{\left(1+\left|\lambda-k^{3}\right|\right)^{b}}{\left(1+\left|\lambda-k^{3}\right|\right)^{b}}|\widehat{u}(k, \lambda)| d \lambda\right)^{2} d k \\
& \leq \delta C_{b}\|u\|_{X_{\gamma, a, b}^{\delta}}^{2} .
\end{aligned}
$$

Therefore,

$$
\|u\|_{X_{\gamma, a, \beta}^{\delta}} \leq C \delta^{\frac{1}{2}\left(1-\frac{\beta}{b}\right)}\|u\|_{X_{\gamma, a, b}^{\delta}} .
$$

The smaller $\beta \in[0, b]$, the larger the power of $\delta$ in (3.9).

Combining (3.7), (3.8) and (3.9) gives

$$
\left\|\int_{0}^{t} S(t-\tau) \partial_{x} u^{2}(\tau) d \tau\right\|_{X_{0, a, b}^{\delta}} \leq C \delta^{\frac{1}{2}\left(1-\frac{\beta}{b}\right)-}\|u\|_{X_{0, a_{1}, b}^{\delta}}\|u\|_{X_{0, a_{2}, b}^{\delta}},
$$

for $b=\frac{1}{2}+,-\frac{3}{4}<a_{i} \leq 0$ and $\frac{1}{4}<\beta<\frac{1}{2}$. Because we want various quantities to depend only upon the $L^{2}$ norm, we set $a_{1}=0$ in (3.10). A contraction estimate will follow if we select $\delta$ so small that

$$
C \delta^{\frac{1}{2}\left(1-\frac{\beta}{b}\right)-}\|u\|_{X_{0,0, b}^{\delta}}<1
$$

As in [1] and [6], we observe that $\|u\|_{X_{0, a, b}^{\delta}} \leq C\left\|\phi_{0}\right\|_{H^{0, a}}$, so we can take $\beta=\frac{1}{4}+$ and choose $\delta$ satisfying (3.3) and still have the contraction estimate.

Proposition 3. The initial value problem (3.2) with initial data $\psi_{0}$ satisfying $\left\|\psi_{0}\right\|_{H^{\sigma, \tilde{a}}} \ll 1$ with $-\frac{1}{2}<\sigma$ and $-\frac{3}{4}<\tilde{a} \leq 0$ and variable coefficient $u \in X_{0, a, b}$ is wellposed in a time interval $[0, \delta]$ where $\delta$ satisfies

$$
\delta^{\frac{1}{4}-}\|u\|_{X_{0,0, b}} \sim 1 .
$$

Moreover, the solution $v$ satisfies

$$
\|v\|_{X_{\sigma, \tilde{a}, b}^{\delta}} \leq C\left\|\psi_{0}\right\|_{H^{\sigma, \tilde{a}}}, b=\frac{1}{2}+.
$$

Proof. We show there exists a function $v \in X_{\sigma, \tilde{a}, b}^{\delta}$ which satisfies for $t \in[0, \delta]$ the integral equation

$$
v_{0}(t)=S(t) \psi_{0}-\int_{0}^{t} S(t-\tau) \frac{1}{2} \partial_{x}\left(v^{2}(\tau)+2 u(\tau) v(\tau)\right) d \tau .
$$

As in the treatment of (3.1), the issues are determined by the bilinear estimate. From Theorem 2, we have

$$
\left\|\partial_{x} v^{2}\right\|_{X_{\sigma, \tilde{a}, b-1}} \leq C\|v\|_{X_{\sigma, \tilde{a}, b}}\|v\|_{X_{\sigma, \tilde{a}, \beta}}
$$


provided $b=\frac{1}{2}+, \frac{1}{4}<\beta<\frac{1}{2},-\frac{3}{4}<\tilde{a} \leq 0$ and $-\sigma<3 \beta-\frac{3}{4}$. As in the earlier discussion, we obtain

$$
\begin{aligned}
\left\|\int_{0}^{t} S(t-\tau) \partial_{x} v^{2}(\tau) d \tau\right\|_{X_{\sigma, \tilde{a}, b}^{\delta}} \leq C \delta^{\frac{1}{2}\left(1-\frac{\beta}{b}\right)}\|v\|_{X_{\sigma, \tilde{a}, b}^{\delta}}\|v\|_{X_{\sigma, \tilde{a}, b}} & \\
& \leq C\|v\|_{X_{\sigma, \tilde{a}, b}^{\delta}}\|v\|_{X_{\sigma, \tilde{a}, b}^{\delta}} .
\end{aligned}
$$

(For this estimate, we take $\beta$ near $\frac{1}{2}$ to push $\sigma$ down near $-\frac{3}{4}$.)

The other term in (3.13) is estimated using Theorem 2,

$$
\left\|\partial_{x} u v\right\|_{X_{\sigma, \tilde{a}, b-1}^{\delta}} \leq C\left(\|u\|_{X_{0,0, b}^{\delta}}\|v\|_{X_{\sigma, \tilde{a}, \beta}^{\delta}}+\|u\|_{X_{0,0, \beta}^{\delta}}\|v\|_{X_{\sigma, \tilde{a}, b}^{\delta}}\right),
$$

provided $b=\frac{1}{2}+, \frac{1}{4}<\beta<\frac{1}{2},-\sigma<\min \left(\frac{3}{2}+2 \tilde{a}, 2 \beta+\frac{1}{2}\right)$. Ultimately, we obtain

$$
\left\|\int_{0}^{t} S(t-\tau) \partial_{x}(u(\tau) v(\tau)) d \tau\right\|_{X_{\sigma, \tilde{a}, b}^{\delta}} \leq C \delta^{\frac{1}{2}\left(1-\frac{\beta}{b}\right)-}\|u\|_{X_{0,0, b}^{\delta}}\|v\|_{X_{\sigma, \tilde{a}, b}^{\delta}} .
$$

(Here, we take $\beta$ near $\frac{1}{4}$ to improve the power of $\delta$.)

A contraction estimate follows provided

$$
\max \left(C\|v\|_{X_{\sigma, \tilde{a}, b}^{\delta}}, C \delta^{\frac{1}{2}\left(1-\frac{\beta}{b}\right)}\|u\|_{X_{0,0, b}^{\delta}}\right)<\frac{1}{2} .
$$

The contraction implies $\|v\|_{X_{\sigma, \tilde{a}, b}^{\delta}} \leq\left\|\psi_{0}\right\|_{H^{\sigma, \tilde{a}}}$ and, since $\left\|\psi_{0}\right\|_{H^{\sigma, \tilde{a}}}$ is very small for large $N$, we can replace (3.16) by

$$
\delta^{\frac{1}{2}(1-2 \beta)}\|u\|_{X_{0,0, b}} \sim 1 .
$$

The support properties of $\widehat{\psi_{0}}$ show that $\tilde{a}$ can be take anywhere in $\left(-\frac{3}{4}, 0\right]$. Take $\beta=\frac{1}{4}+$ to observe wellposedness of $(3.2)$ in $H^{\sigma, \tilde{a}}$ on a $\delta$-sized time interval given by (3.11), provided $-\frac{3}{4}<\sigma$.

\section{Global Wellposedness}

We show global wellposedness of the initial value problem

$$
\left\{\begin{array}{c}
\partial_{t} u+\partial_{x}^{3} u+\frac{1}{2} \partial_{x} u^{2}=0 \\
u(0)=\phi_{0} \in H^{s, a}(\mathbb{R}), \text { for certain } s, a<0 .
\end{array}\right.
$$

It suffices to construct the solution on $[0, T]$ for arbitrary $T \gg 1$. Fix $T$. We construct the solution $u$ of (4.1) as

$$
u(t)=\chi_{[\delta(j-1), \delta j]}(t)\left\{u_{j}(t-\delta(j-1))+v_{j}(t-\delta(j-1))\right\}
$$

where $u_{j}$ and $v_{j}$ solve the initial value problems

$$
\begin{gathered}
\left\{\partial_{t} u_{j}+\partial_{x}^{3} u_{j}+\frac{1}{2} \partial_{x} u_{j}^{2}=0, u_{j}(0)=\phi_{j},\right. \\
\left\{\partial_{t} v_{j}+\partial_{x}^{3} v_{j}+\frac{1}{2} \partial_{x}\left(v_{j}^{2}+2 u_{j}(x, t) v_{j}\right)=0, v_{j}(0)=\psi_{j},\right.
\end{gathered}
$$

for $j=1,2, \ldots,\left[\frac{T}{\delta}+1\right]$. Note that $u_{j}+v_{j}$ solves (4.1) with data $\phi_{j}+\psi_{j}$ if $u_{j}, v_{j}$ solve (4.3), (4.4), respectively. To carry out this construction, we define initial data $\phi_{j}, \psi_{j}$ which allow us to show (4.3), (4.4) are both locally wellposed 
on a $\delta_{j}$-sized time interval determined by $\left\|\phi_{j}\right\|_{L^{2}}$. Observe that (4.4) depends upon $\phi_{j}$ through the coefficient $u_{j}(x, t)$, the solution of (4.3). Moreover, the data may be selected so that

$$
\sup _{j}\left\|\phi_{j}\right\|_{L^{2}} \leq C \inf _{j}\left\|\phi_{j}\right\|_{L^{2}}
$$

allowing us to conclude $\delta_{j}=\delta>0$ for all $j$.

For $\phi \in H^{s, a}$, write $\phi=\phi_{0}+\psi_{0}, \phi_{0}=\mathbb{P}_{N} \phi$. This defines the data $\phi_{0}, \psi_{0}$ for (4.3), (4.4) with $j=0$. Note that

$$
\begin{aligned}
\left\|\phi_{0}\right\|_{L^{2}} & \sim N^{-s}, \\
\left\|\psi_{0}\right\|_{H^{\sigma, \tilde{a}}} & \sim N^{\sigma-s},
\end{aligned}
$$

for arbitrary $\tilde{a}>-\frac{3}{4}$.

The local wellposedness result in Proposition 2 shows $\phi_{0} \longmapsto u_{0}(t)$ is defined for $t \in[0, \delta]$ where

$$
\delta^{\frac{1}{4}-}\left\|\phi_{0}\right\|_{L^{2}} \sim 1
$$

The smallness of $\psi_{0} \in H^{\sigma, \tilde{a}}$ makes the cross term the dominant term in (4.4). As shown in Proposition 3, the evolution $\psi_{0} \longmapsto v_{0}(t)$ is defined for $t \in[0, \delta]$ satisfying (4.8).

Following Bourgain [3], we define

$$
\begin{aligned}
& \phi_{1}=u_{0}(\delta)+w_{0}(\delta) \\
& \psi_{1}=S(\delta) \psi_{0}
\end{aligned}
$$

where $w_{0}(\delta)$ is defined via $v_{0}(t)=S(t) \psi_{0}+w_{0}(t)$. Unitarity of $S(\cdot)$ ensures that $\psi_{1}$ satisfies (4.7). To verify (4.5) for $j=1$ it suffices to show

$$
\left\|\phi_{1}\right\|_{L^{2}} \sim N^{-s}+o\left(N^{-s}\right),
$$

in light of (4.6).

Estimating $\left\|\phi_{1}\right\|_{L^{2}}$. We have

$$
\left\|\phi_{1}\right\|_{L^{2}} \leq\left\|u_{0}(\delta)\right\|_{L^{2}}+\sup _{t \in[0, \delta]}\left\|w_{0}(t)\right\|_{L^{2}}
$$

and by $L^{2}$ conservation of the evolution $\phi_{0} \longmapsto u_{0}(t)$ and properties of $X_{s, a, b}$ spaces

$$
\left\|\phi_{1}\right\|_{L^{2}} \leq\left\|\phi_{0}\right\|_{L^{2}}+\left\|w_{0}\right\|_{X_{0,0, b}} .
$$

Since $v_{0}(t)=S(t) \psi_{0}+w_{0}(t)$, we recognize that

$$
w_{0}(t)=-\int_{0}^{t} S(t-\tau) \frac{1}{2} \partial_{x}\left(v_{0}^{2}(\tau)+2 u_{0}(\tau) v_{0}(\tau)\right) d \tau .
$$

Hence,

$$
\left\|w_{0}\right\|_{X_{0,0, b}} \leq C \delta^{-\epsilon_{b}}\left\|\partial_{x} u_{0} v_{0}\right\|_{X_{0,0, b-1}}+C \delta^{-\epsilon_{b}}\left\|\partial_{x} v_{0}^{2}\right\|_{X_{0,0, b-1}}
$$


By the bilinear estimate, we have

$$
\left\|\partial_{x} u_{0} v_{0}\right\|_{X_{0,0, b-1}} \leq C\left(\left\|u_{0}\right\|_{X_{0, a, b}}\left\|v_{0}\right\|_{X_{\sigma_{1}, a_{1}, \beta}}+\left\|u_{0}\right\|_{X_{0, a, \beta}}\left\|v_{0}\right\|_{X_{\sigma_{1}, a_{1}, b}}\right)
$$

where

$$
-\sigma_{1}<\min \left(2 \beta-\frac{1}{2},-a \frac{2 \beta-\frac{1}{2}}{\beta+\frac{1}{2}}, \frac{3}{2}\right) .
$$

Then, taking $\beta=b$ in (4.16) allows us to manufacture a positive power of $\delta$ to kill $\delta^{-\epsilon_{b}}$. So, we require $-\sigma_{1}<\min \left(\frac{1}{2},-\frac{a}{2}\right)$. Similar arguments apply to the second term in (4.15) allowing us to show

$$
\left\|w_{0}\right\|_{X_{0,0, b}} \leq C\left\{\left\|u_{0}\right\|_{X_{0, a, b}}\left\|v_{0}\right\|_{X_{\sigma_{1}, a_{1}, b}}+\left\|v_{0}\right\|_{X_{\sigma_{2}, a_{2}, b}}^{2}\right\}
$$

for

$$
-\sigma_{2} \leq \min \left(2 \beta-\frac{1}{2},-a_{2} \frac{2 \beta-\frac{1}{2}}{\beta+\frac{1}{2}}, \frac{3}{4}, \beta+\frac{1}{4}, \frac{3}{2} \beta-\frac{3}{8}\right) \leq \min \left(-\frac{a_{2}}{2}, \frac{3}{8}\right)
$$

upon choosing $\beta=b \sim \frac{1}{2}$. The local wellposedness result (Proposition 3) shows the quantities on the right-side of (4.18) are controlled by norms on the initial data $\phi_{0}, \psi_{0}$. Indeed, we have

$$
\left\|w_{0}\right\|_{X_{0,0, b}} \leq C\left\{\left\|\phi_{0}\right\|_{H^{0, a}}\left\|\psi_{0}\right\|_{H^{\sigma_{1}, a_{1}}}+\left\|\psi_{0}\right\|_{H^{\sigma_{2}, a_{2}}}^{2}\right\} .
$$

The definition of $\phi_{0}, \psi_{0}$ then imply

$$
\left\|w_{0}\right\|_{X_{0,0, b}} \leq O\left(N^{\sigma_{1}-2 s}\right) .
$$

Returning to (4.13), we have a quantified version of (4.11),

$$
\left\|\phi_{1}\right\|_{L^{2}} \leq\left\|\phi_{0}\right\|_{L^{2}}+O\left(N^{\sigma_{1}-2 s}\right) .
$$

The appearance of $\left\|\phi_{0}\right\|_{H^{0, a}}$ in (4.19) forecasts the need to estimate $\left\|\phi_{1}\right\|_{H^{0, a}}$ during the next step. So, we estimate this quantity next. Since $H^{0, a} \sim L^{2}+$ $\dot{H}_{|k|<1}^{a}$ and the $L^{2}$ norm is controlled in (4.21), we need to control the contribution arising from the low frequencies. Let $\mathbb{P}$ be the Dirichlet projection onto $|k|<1, \widehat{\mathbb{P f}}(k)=\chi_{\{|k|<1\}}(k) \widehat{f}(k)$. We must estimate

$$
\begin{aligned}
& \left\|\mathbb{P} \phi_{1}\right\|_{H^{0, a}} \leq \\
& \quad\left\|\mathbb{P} S(\delta) \phi_{0}\right\|_{H^{0, a}}+\left\|\mathbb{P} \int_{0}^{\delta} S(\delta-\tau) \frac{1}{2} \partial_{x} u_{0}^{2}(\tau) d \tau\right\|_{H^{0, a}}+\left\|\mathbb{P} w_{0}(\delta)\right\|_{H^{0, a}} .
\end{aligned}
$$

The last term may be estimated as above,

$$
\begin{aligned}
\left\|\mathbb{P} w_{0}(\delta)\right\|_{H^{0, a}} \leq\left\|w_{0}(\delta)\right\|_{H^{0, a}} & \leq\left\|w_{0}\right\|_{X_{0, a, b}} \\
& \leq C\left\{\left\|\phi_{0}\right\|_{H^{0, a}}\left\|\psi_{0}\right\|_{H^{\sigma_{1}, a_{1}}}+\left\|\psi_{0}\right\|_{H^{\sigma_{2}, a_{2}}}^{2}\right\} .
\end{aligned}
$$

The first term in (4.22) is bounded by $\left\|\mathbb{P} \phi_{0}\right\|_{H^{0, a}}$ using the unitarity of $S(\cdot)$ and the commutativity of $\mathbb{P}$ and $S(\cdot)$. The dominant term in (4.22) is controlled using the following lemma. 
Lemma 4. For $a>-\frac{3}{2}$,

$$
\left\|\mathbb{P} \int_{0}^{\delta} S(\delta-\tau) \frac{1}{2} \partial_{x} u_{0}^{2}(\tau) d \tau\right\|_{H^{0, a}} \leq C \delta \sup _{t \in[0, \delta]}\left\|u_{0}(t)\right\|_{L_{x}^{2}}^{2} .
$$

Proof. The left-side is controlled by

$$
\begin{aligned}
C \delta \sup _{t \in[0, \delta]}\left\|\mathbb{P} \partial_{x} u_{0}^{2}(t)\right\|_{H^{0, a}} \\
=C \delta \sup _{t \in[0, \delta]}\left(\int_{|k|<1}|k|^{2(1+a)}\left|\widehat{u_{0}(t)} * \widehat{u_{0}(t)}\right|^{2}(k) d k\right)^{\frac{1}{2}} .
\end{aligned}
$$

Since $a>-\frac{3}{2}$, matters are reduced to controlling

$$
C \delta \sup _{t \in[0, \delta]}\left\|\widehat{u_{0}(t)} * \widehat{u_{0}(t)}\right\|_{L_{x}^{\infty}}
$$

The desired estimate follows from Young's inequality and the Plancherel Theorem.

Combining the estimates on the terms in (4.22) gives the estimate

$$
\left\|\phi_{1}\right\|_{H^{0, a}} \leq C \delta\left\|\phi_{0}\right\|_{L^{2}}^{2}+O\left(N^{-s}\right) \text {. }
$$

We complete the construction of $u$, the solution of (4.1), with an induction argument. Suppose for $j=0,1, \ldots, n$ we write

$$
\begin{gathered}
\phi_{j+1}=u_{j}(\delta)+w_{j}(\delta) \\
\psi_{j+1}=S(\delta) \psi_{j}
\end{gathered}
$$

where $\phi_{j} \longmapsto u_{j}(t), \psi_{j} \longmapsto v_{j}(t)$ are solutions of (4.3), (4.4) for $t \in[0, \delta]$ where $\delta$ satisfies (4.8). Suppose furthermore that

$$
\begin{gathered}
\left\|\phi_{j}\right\|_{L^{2}} \leq\left\|\phi_{0}\right\|_{L^{2}}+T C j N^{\sigma_{1}-3 s} \\
\left\|\phi_{j}\right\|_{H^{0, a}} \leq C j \delta N^{-2 s} .
\end{gathered}
$$

Observe that (4.21) and (4.27) show (4.30) and (4.31) hold for $j=1$. If $n>$ $\left[\frac{T}{\delta}+1\right]$, the solution is constructed on $[0, T]$ and we are done. Therefore, assume $n<\frac{T}{\delta}$. Then $\phi_{n+1}, \psi_{n+1}$ are defined by (4.28), (4.29). We verify (4.30), (4.31) for $j=n+1$.

We have

$$
\left\|\phi_{n+1}\right\|_{L^{2}} \leq\left\|\phi_{n}\right\|_{L^{2}}+C \delta^{-\epsilon_{b}}\left(\left\|\partial_{x} u_{n} v_{n}\right\|_{X_{0,0, b-1}}+\left\|\partial_{x} v_{n}^{2}\right\|_{X_{0,0, b-1}}\right) .
$$

The hypothesis (4.30) and the bilinear estimate (as used in (4.16)-(4.18)) show

$$
\begin{aligned}
& \left\|\phi_{n+1}\right\|_{L^{2}} \leq \\
& \left\|\phi_{0}\right\|_{L^{2}}+T C n N^{\sigma_{1}-3 s}+C\left(\left\|u_{n}\right\|_{X_{0, a, b}}\left\|v_{n}\right\|_{X_{\sigma_{1}, a_{1}, b}}+\left\|v_{n}\right\|_{X_{\sigma_{2}, a_{2}, b}}^{2}\right)
\end{aligned}
$$


provided

$$
-\sigma_{2} \leq \min \left(\frac{3}{8},-\frac{a_{1}}{2}\right),-\sigma_{1} \leq \min \left(\frac{1}{2},-\frac{a}{2}\right) .
$$

By the local result,

$$
\begin{aligned}
& \left\|\phi_{n+1}\right\|_{L^{2}} \leq \\
& \quad\left\|\phi_{0}\right\|_{L^{2}}+T C n N^{\sigma_{1}-3 s}+C\left(\left\|\phi_{n}\right\|_{H^{0, a}}\left\|\psi_{n}\right\|_{H^{\sigma_{1}, a_{1}}}+\left\|\psi_{n}\right\|_{H^{\sigma_{2}, a_{2}}}^{2}\right)
\end{aligned}
$$

and by induction

$$
\left\|\phi_{n+1}\right\|_{L^{2}} \leq\left\|\phi_{0}\right\|_{L^{2}}+T C n N^{\sigma_{1}-3 s}+C\left(n \delta N^{\sigma_{1}-3 s}+N^{2\left(\sigma_{2}-s\right)}\right) .
$$

Since $n \delta<T$, if we take $\sigma_{2}=\frac{\sigma_{1}}{2}, a=a_{2}$, for large $N$, we can write

$$
\left\|\phi_{n+1}\right\|_{L^{2}} \leq\left\|\phi_{0}\right\|_{L^{2}}+T C(n+1) N^{\sigma_{1}-3 s} .
$$

This is the $n+1$ case of (4.30) as desired. The estimate (4.31) follows from the argument (4.21)-(4.26) .

Finally, the uniformity of $\delta$ with respect to $j$ is guaranteed if

$$
\sup _{j}\left\|\phi_{j}\right\|_{L^{2}} \leq 2\left\|\phi_{0}\right\|_{L^{2}} \text {. }
$$

Estimate (4.30) shows we can take $j=1, \ldots, M$ steps with the uniformly sized $\delta$ determined by (4.8) where $M$ satisfies $T C M N^{\sigma_{1}-3 s} \sim N^{-s}$, that is

$$
M \sim \frac{1}{T C} N^{2 s-\sigma_{1}}
$$

This process extends the solution $u$ of $(4.1)$ to the time interval $[0, M \delta]$. By (4.8), (4.6) and (4.39),

$$
M \delta \sim C N^{6 s-\sigma_{1}}
$$

and we can ensure $M \delta>T$ by choice of $N$ if $6 s-\sigma_{1}>0$. Recalling the condition (4.34), we have global wellposedness provided

$$
s>\frac{1}{6} \sigma_{1}=-\frac{1}{6} \min \left(\frac{1}{2},-\frac{a}{2}\right)=\frac{a}{12} .
$$

\section{References}

[1] J. Bourgain, Fourier transform restriction phenomena for certain lattice subsets and applications to nonlinear evolution equations I,II, Geom. Funct. Anal. 3 (1993), 107-156, 209-262.

[2] J. Bourgain, New global wellposedness results for nonlinear Schrödinger equations, preprint, 1998.

[3] J. Bourgain, Refinements of Strichartz' inequality and applications to 2D-NLS with critical nonlinearity, Internat. Math. Res. Notices 5 (1998), 253-283.

[4] G. Fonseca, F. Linares, and G. Ponce, Global well-posedness for the modified Korteweg-de Vries equation, Comm. Partial Differential Equations, 24 (1999), 683-705.

[5] M. Keel and T. Tao, Local and Global Well-Posedness of Wave Maps on $\mathbb{R}^{1+1}$ for Rough Data, Internat. Math. Res. Notices, 21 (1998), 1117-1156. 
[6] C. Kenig, G. Ponce, and L. Vega, A bilinear estimate with applications to the KdV equation, J. Amer. Math. Soc. 9 (1996), 573-603.

[7] H. Takaoka, Global Well-posedness for the Kadomtsev-Petviashvili ii Equation, preprint (to appear in Discrete Cont. Dynamical Systems), 1999.

[8] N. Tzvetkov, Global low regularity solutions for Kadomtsev-Petviashvili equation preprint (to appear in Differential and Integral Equations), 1999.

Department of Mathematics, University of California, Berkeley, CA 94720

E-mail address: colliand@math.berkeley.edu

Department of Mathematics, Stanford University, Stanford, CA 94305

E-mail address: gigliola@math.stanford.edu

Mathematical Institute, Tohoku University, Sendai, 980-8578, JAPAN

E-mail address: k99d99@math.tohoku.ac.jp 